\title{
Identification of Brain Tumor using MATLAB
}

\author{
G Aswani $^{1}$ | I N V V A M S N Murthy ${ }^{1} \mid$ K Durga Devi $^{1}$ | N Veerababu ${ }^{1}$ | M L N Swamy ${ }^{1}$ \\ ${ }^{1}$ Department of ECE, VSM College of Engineering, Ramachandrapuram, AP, India.
}

\section{To Cite this Article}

G Aswani, I N V V A M S N Murthy, K Durga Devi, N Veerababu and M L N Swamy, "Identification of Brain Tumor using MATLAB", International Journal for Modern Trends in Science and Technology, Vol. 06, Issue 06, June 2020, pp.:46-49; https://doi.org/10.46501/IJMTST060611

\section{Article Info}

Received on 28-April-2020, Revised on 20-May-2020, Accepted on 25-May-2020, Published on 04-June-2020.

\section{ABSTRACT}

Brain Tumor detection and removal is one medical issue that still remains challenging in the field of biomedicine. MRI is most often used for the detection of tumors, lesions, and other abnormalities in soft tissues, such as the brain. This project is about detecting Brain tumors from MRI images using an interface of GUI in Mat lab. Using the GUI, this program can use various combinations of segmentation, filters, and other image processing algorithms to realize the simplest results. Here We start the process by filtering the image with the help of Prewitt horizontal edge-emphasizing filter. The next step for detecting tumors is "watershed pixels." The most important part of this project is that all the Mat lab programs work with GUI "Matlab guide".

KEYWORDS: Brain tumor, GUI matlab, MRI image

Copyright (C) 2014-2020 International Journal for Modern Trends in Science and Technology

DOI: https://doi.org/10.46501/IJMTST060611

\section{INTRODUCTION}

Medical field has extensive use of various scanning like X-rays, CT, MRI and Ultrasound. These techniques are quite useful for providing information on the internal organs. However, there is a gap for obtaining information of the outer body parts which can now be taken care by 3D Scanning technologies. So many research papers of different scanning techniques and 3D scanning has been studied to identify the impact of them and the subsequent research.

The symptoms of brain tumor depend on the tumor size, type and location. Some common symptoms of brain tumor are headache, nausea and vomiting. Changes in speech vision or hearing, change in mood, ability to concentrate and problems with memory and walking.A brain tumor is a primary or secondary type depend on its location.

1.Benign 2.Malignant.

\section{SEGMENTATION:}

Image segmentation may be a commonly used technique in digital image processing and analysis to partition a picture into multiple parts or regions, often supported the characteristics of the pixels in the image. Image segmentation could involve separating foreground from background, or clustering regions of pixels supported similarities in color or shape. For example, a common application of image segmentation in medical imaging is to detect and label pixels in a picture or voxels of a $3 \mathrm{D}$ volume that represent a tumor during a patient's brain or otherorgans. 


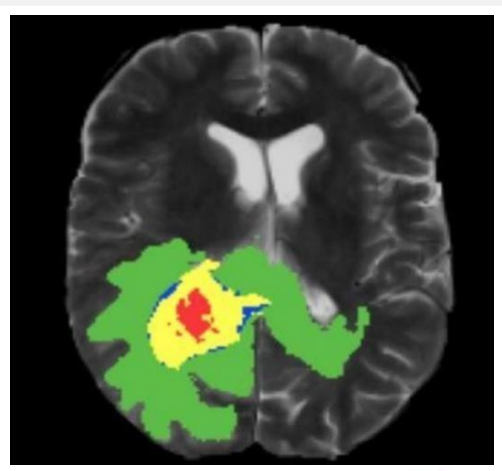

Fig1:Braintumorimagesegmentation

Image segmentation involves converting a picture into a set of regions of pixels that are represented by a mask or a labeled image. By dividing a picture into segments, you'll process only the important segments of the image rather than processing the whole image

\section{Morphological operation:}

Morphology may be a broad set of image processing operations that process images supported shapes. In a morphological operation each pixel within the image is predicated on the worth of the opposite pixels within the neighborhood, we will construct a morphological operation the is sensitive to specific shapes in the input image.

\section{IDENTIFICATION TECHNIQUE CONSISTS OF 3 STAGES}

Step1.Preprocessing(including feature extraction and have reduction); Step 2. coaching the kernel SVM; Step3. Submit new magnetic resonance imaging brains to the trained kernel SVM, and output prediction. This flow diagram may be

a canonical and normal classification technique which has already been tried because the best classification technique. we'll explainthe elaborate $d$ procedures of the pre processing with in the following subsections.

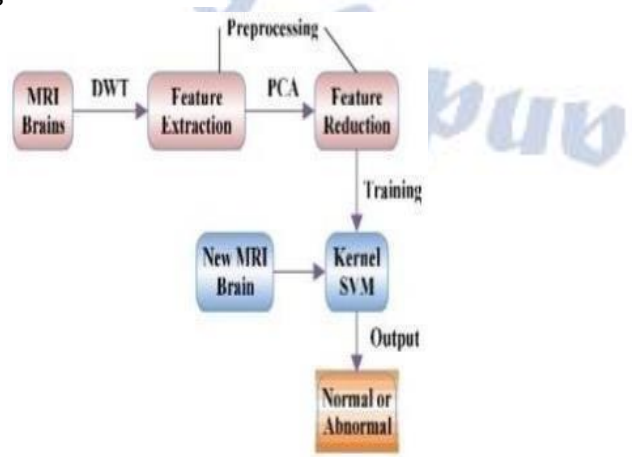

Fig2:Methodology of identification technique

\section{Classification of Accuracy:}

We tested four SVMs with completely different kernels (LIN, HPOL, IPOL, and GRB).In the case of victimisation linear kernel, the KSVM degradesto original linear SVM.We computed many simulations so as to estimate the best parameters of the kernel functions, like the order $d$ in HPOL and IPOL kernel, and also the scaling issue issue in GRB kernel. The confusion matrices of our strategies are listed in Table four. The component of I row and $j$ column represents the category ification accuracy happiness to category $i$ are assigned to class $\mathrm{j}$ when the supervised classification .The results showed that the projected DWT+PCA+KSVM method obtains quite glorious results on each coaching and validation pictures. total classification Classification For carver kernel, the of ten completely different algorithms for a similar magnetic resonance imaging dataset and same variety of pictures.

\section{RESULT}

\section{Mean:}

Mean is most basic of all statistical measure. Means are often used in geometry and analysis; a good range of means are developed for these purposes. In contest of image processing filtering using mean is assessed as spatial filtering and used for noise reduction. In this section we have discussed about various type of mean and analyses their use for removing various type of noise in image processing. Image detail better than the arithmetic mean filter.

\section{Harmonic Mean:}

The harmonic mean filter is yet another variation of the arithmetic mean filter and is useful for images with Gaussian or salt noise. Black pixels (pepper noise) are not filtered. The arithmetic mean filter [2], also known as averaging filter, operates on a sliding ' $\mathrm{m} \times \mathrm{n}$ ' window by calculating the average of all pixel values within the window and replacing the middle pixel value within the destination image with the result. The arithmetic mean filter causes a certain amount of blurring (proportional to the window size) to the image, thereby reducing the consequences of noise and native variations. It can be used to 
reduce noise of different types, but works best for Gaussian, uniform, or Erlanger noise.

\section{Geometric Mean:}

The geometric mean [2] filter is a variation of the arithmetic mean filter and is primarily used on images with Gaussian noise. This filter is understood to retain image detail better than the first

moment filter.

\section{HarmonicMean:}

The harmonic mean filter is yet another variation of the arithmetic mean filter and is useful for images with Gaussian or salt noise. Black pixels (pepper noise) are not filtered.

\section{Contra harmonic Mean}

The contra-harmonic mean filter is another variation of the arithmetic mean filter and is primarily used for filtering salt or pepper noise (but not both). Images with salt noise can be filtered using negative values of $R$, whereas those with pepper noise can be filtered using positive values of $R$.

\section{Median:}

Median is measure of intensity level of pixel which is separating the high intensity value pixels from lower intensity value pixels. It is a type of order-statistic filter. The most popular and useful of the rank filters is the median filter. It works by selecting the center pixel value from the ordered set of values within the ' $m \times n$ ' neighbourhood 'W' round the reference pixel. If ' $\mathrm{mn}$ ' is a good number (which isn't common), the arithmetic average of the 2 values closest to the center of the ordered set is employed instead.

\section{Standard deviation:}

It is a most generally used measure of variability or diversity utilized in statistics. In terms of image processing it shows what proportion variation or "dispersion" exists from the typical (mean, or expected value). a coffee variance indicates that the info points tend to be very on the brink of the mean, whereas high variance indicates that the info points are opened up over an outsized range

\section{Entropy:}

Entropy was introduced by Shannon (1948), were the upper value of Entropy = more detailed information. Entropy may be a measure of image information content, which is interpreted because the average uncertainty of data source. In Image, Entropy is defined as corresponding states of intensity which individual pixels can adapt. it's utilized in the quantitative chemical analysis and evaluation image details; the entropy value is employed because it provides better comparison of the image details. calculate entropy of image: The entropy of a picture are often calculated by calculating at each pixel position $(i, j)$ the entropy of the pixel-values within a 2 -dim region centered at $(\mathrm{I}, \mathrm{j})$. within the following example the entropy of a grey-scale image is calculated and plotted. The region size is configured to be $(2 \mathrm{~N} \times 2 \mathrm{~N})=(10,10)$. RMS:

RMS is that the root-mean-square value of a sign . For a digitized signal, you'll calculate it by squaring each value, finding the first moment of these squared values, and taking the root of the result.

\section{Inverse Difference:}

It is a measure of local homogeneity of a picture . Higher value indicates more homogeneous image. Inverse Difference Moment measures the local homogeneity of a picture. The incidence of cooccurrence of pixel pairs is enhanced once they are draw in gray-scale value and thus increases the IDM value. due to the weighting factor $1 /\left(1+(i-j)^{\wedge} 2\right)$, it'll get small contributions from inhomogeneous areas $i \neq \bar{j}$. The result's a coffee IDM value inhomogeneous images, and a comparatively higher value for homogeneous images..

\section{Contrast image processing:}

Contrast image processing is that the difference in luminance or colour that creates an object distinguishable from other objects within an equivalent field of view a true life example can be of a sunny and a foggy day. On a sunny day, everything looks clear to us, Thus features a high contrast, as compared to a foggy day, where everything looks nearly of an equivalent intensity.
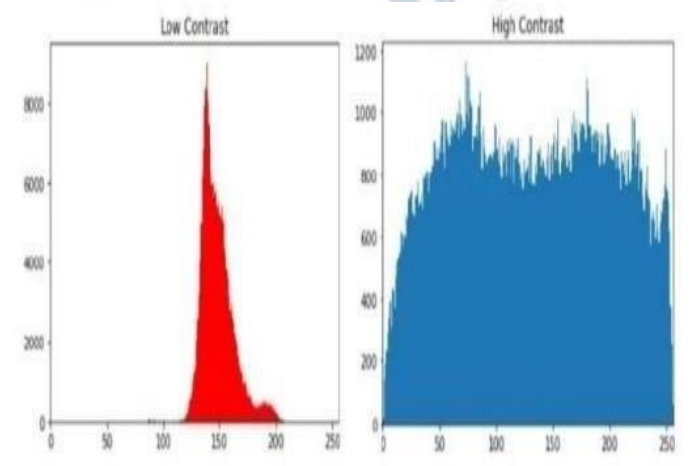

Fig :contrast image processing 


\section{Energy:}

It is used to explain a measure of "information" when formulating an operation under a probability framework like MAP (maximum a priori) estimation in conjunction with Markov Random Fields. Sometimes the energy can be a negative measure to be minimized and sometimes it is a positive measure to be maximized.

\section{Homogeneity:}

Homogeneity measure and a contrast measure defined on the cooccurance matrix of the image.The measure of contrast involves the concept of logarithmic response(adaptability with background intensity) of the human visual system(HVS).

\section{SKEWNESS:}

In terms of digital image processing, Darker and glossier surfaces tend to be more positively skewed than lighter and matte surfaces. Hence we will use skewness in making judgements about image surfaces.

\section{Kurtosis:}

In digital image processing kurtosis values are interpreted together with noise and determination measurement. High kurtosis values should go hand in hand with low noise and low resolution.

\section{CONCLUSION}

A plan for the diagnosis and treatment of brain tumor is a key component of overall control plan. Its main goal is to detect the tumor whether it is non cancerous(benign) or cancerous (malignant). Tumor grow inside the skull and compress the vital structure in the brain,impairing important functions of the brain which can be life threatening. In this we can detected that some of the parameters like mean, variance,standard deviation, skewness, homogeneity etc. As. well as accuracies like RBF accuracy and linear accuracy etc

\section{FUTURE SCOPE:}

Detection of brain tumor is usually based on lot of expenditure. By using this software every individual can determine whether there is

brain tumor cells in the brain by simply using MRI image as input.

\section{REFERENCES}

[1] Y., L. Wu, and S. Wang, "Magnetic resonance brain image classification by an improved artificial bee colony algorithm," Progress In Electromagnetics Research, Vol. 116, 65-79, 2011.
[2] Mohsin, S. A., N. M. Sheikh, and U. Saeed, "MRI induced heating of deepbrain stimulation leads: Effect of the air-tissue interface,"ProgresIn Electromagnetics Research, Vol. 83, 81-91, 2008.

[3] Izquierdo, S. J. Graham, J. R. Mosig, and C. Pollo, "Effect of realistic modeling of deep brain stimulation on the prediction of volume of activated tissue," Progress In Electromagnetics Research, Vol. 126, 1-16, 2012.

[4] Mohsin, S. A., "Concentration of the specific absorption rate around deep brain stimulation electrodes during MRI," Progress In Electromagnetics Research, Vol. 121, 469-484, 2011.

[5] Oikonomou, A., I. S. Karanasiou, and N. K. Uzunoglu, "Phased- array near field radiometry for brain intracranial applications," Progress In Electromagnetics Research, Vol. 109, 345-360,2010.

[6] Scapaticci, R., L. Di Donato, I. Catapano, and L. Crocco, "A feasibility study on microwave imaging for brain stroke monitoring," Progress In Electromagnetics Research B, Vol. 40, 305-324, 2012

[7] Asimakis, N. P., I. S. Karanasiou, P.K. Gkonis, and N. K. Uzunoglu "Theoretical analysis of a passive acoustic brain monitoring system," Progress In Electromagnetics Research B, Vol. 23, 165-180, 2010.

[8] Chaturvedi, C. M., V. P. Singh, P. Singh, P. Basu, M. Singaravel,R. K. Shukla, A. Dhawan, A. K. Pati, R. K. Gangwar, and S. P. Singh. "2.45 GHz (CW) microwave irradiation alters circadian organization, spatial memory, DNA structure in the brain cells and blood cell counts of male mice, mus musculus," Progress Electromagnetics Research B, Vol. 29, 23-42, 2011.

[9] Emin Tagluk, M., M. Akin, and N. Sezgin, "Classification ofsleep apnea by using wavelet transform and artificial neural networks," Expert Systems with Applications, Vol. 37, No. 2, 1600-1607, 2010. 